\title{
Nuovi processi biologici a membrane per la depurazione sostenibile delle acque reflue
}

\author{
Stefano Cairone ${ }^{1}$
}

${ }^{1}$ Affiliation not available

\begin{abstract}
I bioreattori a membrana (MBR) combinano il processo di degradazione biologica a fanghi attivi e il processo fisico di filtrazione con membrane, costituendo un sistema di trattamento avanzato delle acque reflue. Tra i numerosi vantaggi di questa tecnologia, assume particolare importanza l'elevata efficienza depurativa: gli MBR garantiscono la rimozione sia di contaminanti convenzionali che di contaminanti emergenti. Tuttavia, gli MBR presentano degli svantaggi legati principalmente agli elevati costi di investimento e di gestione degli impianti e al controllo del fouling. L'introduzione dei bioreattori a membrana dinamica auto-formante (SFDMBR), basati sull'utilizzo di un materiale di supporto a pori grossolani a basso costo per la formazione di una membrana dinamica auto-formante, consente di ottenere elevate prestazioni depurative a fronte di ridotti costi di investimento. Gli elettro-bioreattori a membrana (e-MBR) combinano processi elettrochimici nei sistemi MBR, con conseguente incremento dell'efficienza depurativa e riduzione del fouling. Combinando la tecnologia SFDMBR con quella e-MBR si ottiene un sistema ibrido estremamente innovativo: l'elettro-bioreattore a membrana dinamica auto-formante (e-SFDMBR).
\end{abstract}

\section{I bioreattori a membrana}

I bioreattori a membrana, noti con l'acronimo MBR, sono impianti per il trattamento avanzato delle acque reflue composti da due parti principali: un bioreattore in cui avviene la biodegradazione dei 
composti organici e un modulo a membrana per la separazione di solidi e microrganismi dall' acqua trattata. I principali vantaggi di questa tecnologia derivano dalla sostituzione del sedimentatore secondario, previsto nel processo biologico a fanghi attivi, con un filtro a membrana ${ }^{1}$ : alla separazione per gravità che si attua nei sedimentatori, che presenta inconvenienti legati all'effettiva capacità dei fiocchi di fango di aggregarsi fra loro e quindi di decantare, viene sostituita una fase di filtrazione con moduli di membrana ${ }^{2}$. In un processo a fanghi attivi convenzionale (CAS) la separazione solido-liquido è ottenuta mediante sedimentazione, che si basa sulla formazione di fiocchi di fango di dimensioni sufficienti $(>50 \mu \mathrm{m})$ a consentire la loro rimozione, mentre in un MBR la separazione solido-liquido avviene per filtrazione, per cui è sufficiente che le particelle siano più grandi della dimensione dei pori della membrana ${ }^{3}$. I bioreattori a membrana sono in grado di rimuovere efficacemente un ampio spettro di microinquinanti organici, inclusi composti resistenti al processo a fanghi attivi, la cui rimozione dipende dalle condizioni del trattamento e dalle proprietà fisico-chimiche delle sostanze ${ }^{4}$. Questo sistema di trattamento avanzato garantisce prestazioni depurative elevate, che comportano una produzione di effluente finale di qualità migliore rispetto al trattamento a fanghi attivi, e svincolate dalla capacità di sedimentazione della biomassa ${ }^{5}$. Grazie all' elevata efficienza di rimozione degli inquinanti e dei microrganismi patogeni, l'effluente trattato potrebbe essere riutilizzato come acqua di recupero ${ }^{6}$. La possibilità di riutilizzare l'acqua trattata con sistemi MBR, incrementando conseguentemente la risorsa idrica disponibile, assume oggigiorno enorme importanza, considerando che la crescita della popolazione e l'industrializzazione stanno causando aumento della domanda d'acqua e contaminazione ${ }^{7}$. I bioreattori a membrana possono essere impiegati sia per realizzare impianti ex novo sia per adeguare impianti esistenti ormai obsoleti o sottodimensionati. Un ulteriore vantaggio è legato alla richiesta di ridotte superfici da occupare: gli MBR sommersi occupano circa la metà della superficie occupata da un impianto a fanghi attivi convenzionale. Inoltre, rispetto a quest'ultimo, la produzione di fanghi è circa dimezzata, il fango resta all'interno del serbatoio di aerazione a contatto con la biomassa più a lungo ${ }^{8}$ e presenta migliori caratteristiche in termini di stabilità e disidratabilità ${ }^{5}$. La possibilità di 
applicare i bioreattori a membrana per la rimozione di composti farmaceutici (non trattabili con sistemi convenzionali) rappresenta un aspetto molto positivo di questa tecnologia. I prodotti farmaceutici, così come altri contaminanti emergenti, sono frequentemente rilevati negli effluenti degli impianti di trattamento delle acque reflue (WWTP) e nelle acque superficiali e potabili a causa del loro carattere idrofilo e della capacità di resistere in ambiente acquatico ${ }^{9}$. Sebbene questi composti siano presenti a bassi livelli di concentrazione nell' ambiente ${ }^{10}$, il loro continuo rilascio dagli WWTP può rappresentare una potenziale minaccia a lungo tempo per gli ecosistemi ${ }^{11}$. Infatti, $\mathrm{i}$ loro impatti negativi non possono essere trascurati poiché possono causare effetti tossici anche a concentrazioni molto basse ${ }^{12}$. L'integrazione dei metodi di trattamento avanzato nei WWTP è essenziale al fine di ostacolare la diffusione dei contaminanti emergenti nell'ambiente in quanto i processi convenzionali non ne consentono una rimozione efficiente ${ }^{13}$.

Nonostante i numerosi aspetti vantaggiosi, l'espansione degli MBR è ostacolata da alcuni fattori negativi, tra i quali il fouling, i maggiori consumi energetici rispetto agli impianti a fanghi attivi convenzionali e l'elevato costo di acquisto della membrana.

\section{Il fouling nei bioreattori a membrana}

A parità di intensità della forza motrice, le condizioni di funzionamento di una membrana non si mantengono costanti nel tempo: le resistenze da vincere aumentano a causa della graduale chiusura dei pori che comporta riduzione della superficie filtrante della membrana ${ }^{5}$. Questo fenomeno, noto come fouling, consiste nello sporcamento della superficie esterna o interna della membrana causato da materiale adsorbito o accumulato su essa durante la filtrazione. Tuttavia, tale termine è usato per indicare tutti i fenomeni che provocano una riduzione della permeabilità delle membrane e che comportano la necessità di superfici filtranti maggiori e pressioni applicate più elevate ${ }^{14}$. A causa delle difficoltà nel controllarlo e nel prevederne gli effetti, il fouling ha fortemente condizionato 
l'applicazione dei bioreattori a membrana. L'accumulo di colloidi, particelle, macromolecole e sali sulla superficie della membrana comporta una diminuzione del flusso con conseguente riduzione della vita utile della membrana ${ }^{15}$. Il fouling è il principale problema operativo degli MBR: la tendenza alla diminuzione della permeabilità della membrana durante il ciclo di filtrazione comporta la richiesta di una periodica pulizia fisica e chimica dei moduli filtranti, con conseguente aumento dei costi di esercizio e problemi legati a tempi di inattività dell'impianto ${ }^{16}$.

Il fouling può essere attribuito sia alla precipitazione di composti inorganici poco solubili, come il carbonato di calcio $\left(\mathrm{CaCO}_{3}\right)$, sia ad inevitabili manifestazioni biologiche, generate da colonie microbiche che interagiscono con la membrana, usandola anche come supporto o come substrato ${ }^{5}$. A seconda delle cause che lo determinano, il fouling può essere distinto in reversibile, irreversibile e irrecuperabile (Fig. 2). Il fouling reversibile può essere efficacemente rimosso con processi fisici, ad esempio con il controlavaggio. Sull'aliquota non eliminabile in questo modo (fouling irreversibile) si agisce ricorrendo, più saltuariamente, alla pulizia chimica utilizzando acidi, basi, tensioattivi, detergenti e ossidanti ${ }^{17}$. Occorre limitare questo tipo di pulizia in quanto frequenti e intensivi lavaggi chimici, oltre ad essere costosi, comportano indebolimento della membrana ${ }^{3}$. Vi sono ulteriori depositi che non possono essere rimossi neanche con pulizia chimica e formano il cosiddetto "fouling irrecuperabile"; queste incrostazioni, accumulandosi nel tempo, comportano la necessità di sostituire la membrana.

Tra i principali precursori del fouling la comunità scientifica individua le Sostanze Polimeriche Extracellulari (EPS), i Prodotti Microbici Solubili (SMP), i biopolimeri e cluster biopolimerici (BPC) e le Particelle Esopolimeriche Trasparenti (TEP). Questi composti sono prodotti ed escreti dai microrganismi; tuttavia ciò che viene analizzato come EPS, SMP, BPC o TEP non è necessariamente di origine microbica, ma può anche essere di natura terrestre o artificiale ${ }^{3}$. In realtà, i parametri che influenzano la formazione del fouling sono vari e sono legati alla tipologia delle membrane im- 
piegate, alle caratteristiche della biomassa presente nel refluo da trattare e alle condizioni operative dell'impianto MBR ${ }^{19}$.

\section{Bioreattori a membrana dinamica auto-formante}

La presenza di consorzi microbici non flocculanti nei processi di trattamento delle acque reflue è stata tradizionalmente considerata un problema ${ }^{20}$. Infatti, la massa filamentosa dei fanghi attivi, il bio-fouling nei bioreattori a membrana (MBR) e l'accumulo di biomassa in reattori non perfettamente miscelati sono considerati tra le principali minacce per le prestazioni ottimali del processo a fanghi attivi ${ }^{21}$. Tuttavia, sono state ideate nuove tecnologie di trattamento delle acque reflue basate sullo sviluppo della biomassa non flocculata ${ }^{20}$, tra cui il bioreattore a membrana dinamica auto-formante (Self-Forming Dynamic Membrane BioReactor, SFDMBR). L'innovazione apportata da questa tecnologia consiste nella sostituzione dei costosi moduli a membrana con una membrana dinamica auto-formante (SFDM). La chiave di tale processo è l'autoformazione di uno strato biologico filtrante (la membrana dinamica, DM) su un supporto di materiale inerte ${ }^{22}$ ad opera delle sostanze presenti nella miscela da filtrare, particolarmente colloidi e sostanze organiche a peso molecolare alto ${ }^{23}$. La formazione della DM è un processo complesso che coinvolge diversi meccanismi fisico-chimici e microbiologici, come deposito di particelle, blocco dei pori e formazione di uno strato biologico, noto come strato di "cake" ${ }^{24}$ (Fig. 3). Tale strato presenta porosità inferiore rispetto al supporto utilizzato per la formazione della membrana, risultando più selettivo rispetto a esso e svolgendo un ruolo chiave nei processi di filtrazione ${ }^{23}$. I processi di formazione di una membrana dinamica non sono immediati e dipendono dalla dimensione dei pori del materiale di supporto, dalle dimensioni delle particelle del fango attivo, dalle caratteristiche del refluo da trattare e dalle condizioni operative.

Diversi studi hanno dimostrato che l'effluente ottenuto dall'impiego di SFDMBR ha bassa qualità 
nelle prime fasi di funzionamento: la filtrazione deve avere una durata sufficiente affinché si possa ottenere un'efficace separazione solido-liquido. Infatti, sebbene la membrana dinamica riesca a trattenere le particelle di fango all'interno del bioreattore e ottenere un'elevata rimozione di solidi sospesi (SS), l'effluente ottenuto nelle fasi iniziali della filtrazione, quando la membrana non è ancora completamente formata, ha qualità generalmente scarsa e può contenere elevate concentrazioni di SS a causa del passaggio di fango attraverso i pori del supporto relativamente grandi. Tuttavia, formata la membrana dinamica, la qualità dell'effluente aumenta gradualmente durante il processo. Si ha riduzione della torbidità dell'effluente nel tempo, che risulta essere un indicatore del processo di microfiltrazione: una significativa riduzione della torbidità in impianti SFDMBR indica che le membrane dinamiche auto-formanti comportano un processo di trattamento simile alla microfiltrazione ${ }^{25}$.

La tecnologia SFDMBR comporta numerosi vantaggi rispetto ai bioreattori a membrana convenzionali, tra cui:

- ridotti costi di investimento;

- migliore controllo del fouling;

- elevato flusso permeato,

- elevate capacità di trattamento nella fase stabile.

La sostituzione dei costosi moduli a membrana con materiali a pori grossolani a basso costo rende gli impianti SFDMBR più convenienti dal punto di vista economico rispetto agli MBR ${ }^{26}$. Solitamente vengono utilizzati supporti in acciaio inossidabile, filtri in tessuto, reti in materiale polimerico e filtri in tessuto non tessuto con dimensioni dei pori comprese tra meno di $10 \mu \mathrm{m}$ e $2 \mathrm{~mm}^{27}$. Tuttavia, analogamente ai moduli a membrana impiegati negli impianti MBR, anche le SFDM sono sottoposte al fenomeno del fouling. Sul materiale di supporto, dopo il deposito iniziale dei componenti necessari per la formazione della membrana dinamica, continuano a depositarsi ulteriori particelle che comportano un aumento eccessivo della resistenza alla filtrazione ${ }^{28}$. A causa dei 
continui processi di deposizione e adsorbimento di fango sulla membrana si formano incrostazioni che comportano un aumento del costo operativo ${ }^{29}$.

\section{Applicazione di processi elettrochimici nei sistemi MBR}

Recentemente, in diversi studi scientifici, sono stati integrati processi elettrochimici nei sistemi MBR per affrontare i problemi relativi al fouling e all' abbattimento dei microinquinanti ${ }^{30}$. Questo sistema ibrido, denominato elettro-bioreattore a membrana (e-MBR), si basa sull'integrazione di impianti MBR con processi elettrochimici (Fig. 4) che, instaurandosi a seguito dell' applicazione di un campo elettrico tramite elettrodi posti nel reattore biologico, migliorano sia le prestazioni depurative che il controllo del fouling ${ }^{31}$. In particolare, i processi elettrochimici comportano una forte riduzione delle concentrazioni di Prodotti Microbici Solubili (SMP) con conseguente diminuzione della propensione al fouling. Inoltre, questa tecnologia incrementa l'efficienza del trattamento delle acque reflue, garantendo eccellenti capacità di rimozione di contaminanti convenzionali ed emergenti, tra cui i prodotti farmaceutici ${ }^{32}$.

L'elettrocoagulazione è il principale processo elettrochimico che si instaura all'interno di un elettro-bioreattore a membrana. Questo processo consente di rimuovere dalle acque reflue diversi contaminanti, come metalli pesanti, solidi sospesi e sostanze organiche, utilizzando corrente elettrica ed elettrodi metallici sacrificali al posto di costosi reagenti chimici. Gli elettrodi utilizzati per l'elettrocoagulazione sono, solitamente, in alluminio e ferro, in quanto risultano essere materiali economici, prontamente disponibili, efficaci e in grado di accelerare il processo di coagulazione ${ }^{34}$. In particolare, l'elettrocoagulazione e la neutralizzazione della carica delle particelle permettono a queste ultime di unirsi, formando fiocchi di maggiori dimensioni ${ }^{35}$. Questi fiocchi potrebbero essere facilmente rimossi con la filtrazione poiché sono relativamente grandi; inoltre contengono meno acqua e sono più stabili ${ }^{36}$. Rispetto alla tradizionale coagulazione chimica, 
l'elettrocoagulazione riduce la produzione di fanghi, non prevede l'uso di agenti chimici costosi e richiede facili procedure operative ${ }^{37}$. Inoltre, ${ }^{38}$ hanno dimostrato la possibilità di utilizzare il processo di elettrocoagulazione come metodo per l'eliminazione dei prodotti farmaceutici dalle acque reflue urbane.

Nell'elettro-bioreattore avvengono diversi processi elettrocinetici che aiutano a ridurre le concentrazioni di sostanze organiche solubili e colloidali ${ }^{39} \cdot{ }^{34}$ distingue due fenomeni elettrocinetici:

- Elettroforesi: movimento di una particella carica attraverso un fluido stazionario sotto l'influenza di un campo elettrico;

- Elettroosmosi: movimento di un liquido rispetto a una superficie carica stazionaria sotto l'influenza di un campo elettrico. Dunque, il fenomeno dell'elettroosmosi riguarda anche la permeazione di un fluido attraverso un mezzo poroso (una membrana).

Nonostante i numerosi aspetti positivi, l'applicazione degli e-MBR presenta alcuni svantaggi. In particolare, gli effetti del campo elettrico sottopongono le specie batteriche presenti nel reattore, responsabili della degradazione biologica, ad un forte shock elettrico che ne inibisce il metabolismo. Di conseguenza, per non influenzare l'attività microbica, necessaria a garantire il processo biologico, i parametri di funzionamento del campo elettrico devono essere calibrati con attenzione. Inoltre, le reazioni che avvengono negli e-MBR comportano corrosione degli elettrodi, con conseguente richiesta di sostituzione degli stessi che porta ad un incremento dei costi operativi.

\section{Modellazione di un elettro-bioreattore a membrana dinamica}

\section{auto-formante}

${ }^{40}$ hanno esaminato per la prima volta la possibilità di combinare processi elettrochimici con bioreattori a membrana dinamica auto-formante (SFDMBR) per la rimozione dei contaminanti emer- 
genti dalle acque reflue. La combinazione di queste due innovative tecniche di depurazione porta alla realizzazione di un elettro-bioreattore a membrana dinamica auto-formante (e-SFDMBR). Tale configurazione, a fronte di ridotti costi di investimento per l'assenza dei costosi moduli a membrana, ha consentito una riduzione del fouling di membrana e un'elevata rimozione di composti organici e nutritivi. Inoltre, i meccanismi elettrochimici sviluppati nel bioreattore hanno consentito una maggiore rimozione dei contaminanti rispetto ai sistemi MBR.

L'evoluzione delle conoscenze in ambito informatico e l'adozione di strumenti tecnologicamente avanzati hanno favorito un continuo miglioramento in molteplici ambiti, tra i quali la progettazione di opere civili ed ambientali. L'avvento di software CAD sempre più avanzati non solo permette la realizzazione di modelli in grado di rappresentare realisticamente il progetto da realizzare, ma consente anche di simulare il funzionamento dell'opera stessa. Utilizzando tali strumenti, sono stati realizzati i modelli virtuali tridimensionali e le simulazioni dei processi di funzionamento dei seguenti impianti:

- Bioreattore a membrana dinamica auto-formante (SFDMBR) a scala di laboratorio;

- Elettro-bioreattore a membrana dinamica auto-formante (e-SFDMBR) a scala di laboratorio;

- Elettro-bioreattore a membrana dinamica auto-formante (e-SFDMBR) a scala pilota, situato presso l'impianto di depurazione "Tavernola" del Comune di Battipaglia (SA).

L'impianto SFDMBR a scala di laboratorio è costituito da un reattore cilindrico in plexiglass avente capienza pari a 19 litri (altezza $=45 \mathrm{~cm}$, diametro $=30 \mathrm{~cm}$, spessore $=10 \mathrm{~mm}$ ), in cui sono stati inseriti dei diffusori a bolle fini collegati ad un compressore per permettere la continua aerazione del sistema. La continua aerazione garantisce le condizioni aerobiche, fornisce una buona miscelazione del refluo ed evita la sedimentazione dei fiocchi di fango. Al centro del reattore è stato posto il materiale di supporto per la formazione della membrana dinamica auto-formante. Come materiale filtrante è stato scelto il dacron, una fibra tessile sintetica in tetrapolietilene ottenuta per condensazione dell' acido tereftalico $\left(\mathrm{C}_{8} \mathrm{H}_{6} \mathrm{O}_{4}\right)$ e del glicol etilenico $\left(\mathrm{C}_{2} \mathrm{H}_{6} \mathrm{O}_{2}\right)$, avente porosità pari 
a $30 \mu \mathrm{m}$. Il dacron è stato inserito all'interno di un supporto in plexiglass, composto da un corpo centrale, in cui è stato posizionato il materiale filtrante, chiuso ermeticamente tramite due telai in plexiglass posizionati esternamente e fissati ad esso con una serie di bulloni. In particolare, si è scelto di posizionare due strati filtranti in dacron per ogni faccia del supporto e, al fine di evitarne l'adesione e per irrigidirli, si è deciso di posizionare tra essi una rete rigida in materiale plastico. La membrana utilizzata presenta dimensioni pari a $6.5 \mathrm{~cm}$ x $16.5 \mathrm{~cm}$, con una superficie filtrante totale pari a $214.5 \mathrm{~cm}^{2}$.

Nell'impianto sono previsti un serbatoio di alimentazione in cui è posto il refluo sintetico utilizzato per alimentare il reattore biologico tramite pompa peristaltica, un serbatoio in cui viene immesso l'effluente prelevato tramite pompa dosatrice e un serbatoio contenente acqua distillata prelevata con pompa peristaltica nella fase di pulizia fisica della membrana (controlavaggio). Per tale sistema è stato previsto un ciclo di funzionamento di 10 minuti, di cui 9 minuti di filtrazione e 1 minuto di controlavaggio. Impostando un flusso pari a $30 \mathrm{LMH}$ si ottiene una portata in ingresso pari a $6.42 \mathrm{ml} / \mathrm{min}$, mentre la portata in uscita è stata impostata a $10.7 \mathrm{ml} / \mathrm{min}$ e la portata di controlavaggio a $32.1 \mathrm{ml} / \mathrm{min}$. Mediante un trasduttore di pressione collegato ad un data logger è possibile monitorare costantemente l'andamento della pressione di transmembrana (TMP) e salvare automaticamente i dati acquisiti in un PC. La TMP, che rappresenta la differenza di pressione tra i due lati della membrana causata dalla resistenza esercitata dalla membrana stessa, è uno dei parametri che deve essere monitorato in quanto fornisce informazioni su come procede la filtrazione. Infatti, a valori elevati della pressione di transmembrana, che potrebbero essere dovuti ad un'eccessiva incrostazione del materiale filtrante, il processo di filtrazione non procede correttamente e ne consegue una necessaria pulizia della membrana.

L'impianto e-SFDMBR a scala di laboratorio è simile a quanto descritto per l'impianto SFDMBR a scala di laboratorio, con l'unica differenza legata all'aggiunta di una coppia di elettrodi cilindrici perforati (un anodo in alluminio dal diametro di $24 \mathrm{~cm}$ e un catodo in acciaio inossidabile dal 
diametro di $12 \mathrm{~cm}$ ) all'interno del bioreattore e collegati ad un generatore di corrente tramite una coppia di morsetti. In particolare, gli elettrodi sono stati posizionati ad una distanza pari a $6 \mathrm{~cm}$, in accordo allo studio svolto da ${ }^{30}$ in cui è sottolineata l'importanza di mantenere una distanza appropriata fra i due elettrodi, allo scopo di minimizzare i potenziali effetti negativi sulla biomassa. La corrente elettrica, caratterizzata da un'intensità pari a $0.5 \mathrm{~mA} / \mathrm{cm}^{2}$, è stata applicata con cicli di 25 minuti (di cui 5 minuti in modalità "ON" e 20 minuti “OFF").

L'impianto e-SFDMBR a scala pilota è situato presso l'impianto di depurazione "Tavernola" del comune di Battipaglia (SA). Dopo essere stato testato in assenza del campo elettrico (SFDMBR pilota), sono stati aggiunti gli elettrodi, ottenendo un elettro-bioreattore a membrana dinamica autoformante. Nel reattore biologico, oltre al supporto per la formazione della SFDM (dimensioni pari a $18 \mathrm{~cm}$ x $30 \mathrm{~cm}$ ), sono stati posti due elettrodi cilindrici perforati (un anodo in alluminio dal diametro di $41 \mathrm{~cm}$ e un catodo in acciaio inossidabile dal diametro di $28.6 \mathrm{~cm}$ ), una serie di diffusori a bolle fini alimentati mediante 5 pompe di aerazione e un interruttore a galleggiante (float switch), utile per rilevare il livello del liquido all'interno del bioreattore. Il refluo utilizzato per alimentare in continuo l'impianto pilota, proveniente dai precedenti trattamenti preliminari di grigliatura, rotostacciatura e dissabbiatura presenti nell'impianto reale, viene immesso nel bioreattore tramite l'azione di una pompa dosatrice; al termine del processo, il refluo trattato (permeato) viene reimmesso in testa al dissabbiatore tramite un'ulteriore pompa dosatrice. Analogamente all'impianto e-SFDMBR a scala pilota, è previsto un ciclo di funzionamento di 10 minuti: 9 minuti di filtrazione e 1 minuto di controlavaggio (in questa fase una pompa dosatrice preleva l'acqua di rete conservata in un apposito serbatoio).

Le principali caratteristiche progettuali definite per l'impianto e-SFDMBR a scala pilota sono riportate in figura 5. 


\section{Conclusioni}

I bioreattori a membrana rappresentano una valida alternativa ai convenzionali impianti di depurazione a fanghi attivi. L'effluente depurato con questa tecnologia può essere considerato come una risorsa riutilizzabile, in quanto riesce a soddisfare i parametri richiesti dalle normative vigenti per il riutilizzo, perseguendo i principi di sviluppo sostenibile. Inoltre, gli ingombri ridotti rendono gli MBR una soluzione interessante per l'adeguamento di impianti di depurazione esistenti, oltre che per la realizzazione di nuovi impianti. Nonostante gli innumerevoli vantaggi presentati da questa tecnologia, tra cui la capacità di rimozione dei contaminanti emergenti, la diffusione dei bioreattori a membrana è ostacolata prevalentemente dagli elevati costi di investimento e di gestione degli impianti, dovuti principalmente al fouling.

L'applicazione dei bioreattori a membrana dinamica auto-formante (SFDMBR) garantisce elevate performance depurative a fronte di ridotti costi di investimento. Questa tecnologia prevede l'utilizzo di un materiale di supporto a pori grossolani a basso costo per la formazione di una membrana dinamica auto-formante (SFDM) che comporta una notevole riduzione delle spese legate all'acquisto degli onerosi moduli a membrana convenzionali.

Al fine di incrementare le efficienze di depurazione e mitigare gli effetti del fouling, l'applicazione di processi elettrochimici all'interno del bioreattore nei sistemi MBR risulta essere un'ottima soluzione. Infatti, i processi elettrochimici, sviluppati a seguito dell'introduzione di un campo elettrico nel bioreattore, riducono la propensione al fouling e garantiscono una maggiore capacità di rimozione dei contaminanti.

Dalla combinazione di queste tecnologie si ottiene un elettro-bioreattore a membrana dinamica auto-formante (e-SFDMBR) che rappresenta una tecnologia non del tutto sviluppata, se non dal Laboratorio di Ingegneria Sanitaria Ambientale (SEED) dell’Università degli Studi di Salerno. I risultati ottenuti studiando gli e-SFDMBR favoriscono la possibilità di introdurre tale brillante 
innovazione anche in impianti a scala pilota per verificarne l'applicabilità in impianti a scala reale.

Lo studio condotto ha permesso anche di evidenziare l'importanza dell'adozione di software di progettazione avanzati che favoriscono un continuo miglioramento in vari ambiti, tra cui la progettazione di opere civili ed ambientali. La realizzazione di modelli virtuali tridimensionali di impianti SFDMBR ed e-SFDMBR a scala di laboratorio e a scala pilota, oltre a garantire una rappresentazione realistica di tali impianti, ha reso possibile eseguirne simulazioni del funzionamento. Sono stati ottenuti elaborati multimediali che mostrano i processi di trattamento delle acque reflue dovuti all'applicazione di queste promettenti tecnologie, impiegate per una depurazione che miri a uno sviluppo sostenibile a salvaguardia dell'uomo e dell'ambiente e che al tempo stesso riduca drasticamente i costi della depurazione.

\section{References}

1.Ahmed, F. N. \& Lan, C. Q. Treatment of landfill leachate using membrane bioreactors: A review. Desalination 287, 41-54 (2012).

2.Viviani, G. Criteri di progettazione dei bioreattori a membrana. in Bioreattori a membrana per la depurazione delle acque reflue (2012).

3.Judd, S. The status of membrane bioreactor technology. Trends in Biotechnology 26, 109-116 (2008).

4.Krzeminski, P., Leverette, L., Malamis, S. \& Katsou, E. Membrane bioreactors - A review on recent developments in energy reduction fouling control, novel configurations, LCA and market prospects. Journal of Membrane Science 527, 207-227 (2017).

5.Pirozzi, F. Principi di funzionamento e configurazioni degli MBR. in Bioreattori a membrana per la depurazione delle acque reflue (2012). 
6.Yin, X., Li, X., Hua, Z. \& Ren, Y. The growth process of the cake layer and membrane fouling alleviation mechanism in a MBR assisted with the self-generated electric field. Water Research 171, 115452 (2020).

7.Gündoğdu, M., Jarma, Y. A., Kabay, N., Pek, T. Ö. \& Yüksel, M. Integration of MBR with $\mathrm{NF} / \mathrm{RO}$ processes for industrial wastewater reclamation and water reuse-effect of membrane type on product water quality. Journal of Water Process Engineering 29, 100574 (2019).

8.Gander, M., Jefferson, B. \& Judd, S. Aerobic MBRs for domestic wastewater treatment: a review with cost considerations. Separation and Purification Technology 18, 119-130 (2000).

9. Verlicchi, P. \& Zambello, E. Pharmaceuticals and personal care products in untreated and treated sewage sludge: Occurrence and environmental risk in the case of application on soil - A critical review. Science of The Total Environment 538, 750-767 (2015).

10.Koba, O. et al.. Transport of pharmaceuticals and their metabolites between water and sediments as a further potential exposure for aquatic organisms. Journal of Hazardous Materials $\mathbf{3 4 2}$, 401-407 (2018).

11.Zhang, D. et al.. Characterization of soluble microbial products (SMPs) in a membrane bioreactor (MBR) treating synthetic wastewater containing pharmaceutical compounds. Water Research 102, 594-606 (2016).

12.Liu, J. L. \& Wong, M. H. Pharmaceuticals and personal care products (PPCPs): A review on environmental contamination in China. Environment International 59, 208-224 (2013).

13.Naddeo, V. et al.. Removal of contaminants of emerging concern from real wastewater by an innovative hybrid membrane process - UltraSound Adsorption and Membrane ultrafiltration (USAMe®). Ultrasonics Sonochemistry 68, 105237 (2020).

14.Ricco, D., Naddeo, V. \& Belgiorno, V. Il ruolo dei polisaccaridi nella formazione del fouling. 
in Bioreattori a membrana per la depurazione delle acque reflue (2012).

15.Borea, L. et al.. Wastewater treatment by membrane ultrafiltration enhanced with ultrasound: Effect of membrane flux and ultrasonic frequency. Ultrasonics 83, 42-47 (2018).

16.Buzatu, P. et al.. Clogging vs. fouling in immersed membrane bioreactors. Water Research 144, 46-54 (2018).

17.Wang, Z. et al. Membrane cleaning in membrane bioreactors: A review. Journal of Membrane Science 468, 276-307 (2014).

18.Meng, F. et al.. Recent advances in membrane bioreactors (MBRs): Membrane fouling and membrane material. Water Research 43, 1489-1512 (2009).

19.Le-Clech, P., Chen, V. \& Fane, T. A. G. Fouling in membrane bioreactors used in wastewater treatment. Journal of Membrane Science 284, 17-53 (2006).

20.Vergine, P., Salerno, C., Berardi, G. \& Pollice, A. Sludge cake and biofilm formation as valuable tools in wastewater treatment by coupling Integrated Fixed-film Activated Sludge (IFAS) with Self Forming Dynamic Membrane BioReactors (SFD-MBR). Bioresource Technology 268, 121-127 (2018).

21.Judd, S. \& Judd, C. The MBR Book - Second Edition: Principles and Applications of Membrane Bioreactors in Water and Wastewater Treatment. (Elsevier, 2011).

22.Salerno, C., Vergine, P., Berardi, G. \& Pollice, A. Influence of air scouring on the performance of a Self Forming Dynamic Membrane BioReactor (SFD MBR) for municipal wastewater treatment. Bioresource Technology 223, 301-306 (2017).

23.Fan, B. \& Huang, X. Characteristics of a Self-Forming Dynamic Membrane Coupled with a Bioreactor for Municipal Wastewater Treatment. Environmental Science \& Technology 36, $5245-5251$ (2002). 
24.Bae, T. H. \& Tak, T. M. Interpretation of fouling characteristics of ultrafiltration membranes during the filtration of membrane bioreactor mixed liquor. Journal of Membrane Science 264, $151-160(2005)$.

25.Rezvani, F., Mehrnia, M. R. \& Poostchi, A. A. Optimal operating strategies of SFDM formation for MBR application. Separation and Purification Technology 124, 124-133 (2014).

26.Wu, Y., Huang, X., Wen, X. \& Chen, F. Function of dynamic membrane in self-forming dynamic membrane coupled bioreactor. Water Science and Technology 51, 107-114 (2005).

27.Mohan, S. M. \& Nagalakshmi, S. A review on aerobic self-forming dynamic membrane bioreactor: Formation performance, fouling and cleaning. Journal of Water Process Engineering 37, 101541 (2020).

28.Chu, H. et al.. Dynamic membrane bioreactor for wastewater treatment: Operation critical flux, and dynamic membrane structure. Journal of Membrane Science 450, 265-271 (2014).

29.Liang, S. et al.. Determination of fouling-related critical flux in self-forming dynamic membrane bioreactors: Interference of membrane compressibility. Journal of Membrane Science 390391, 113-120 (2012).

30.Borea, L., Naddeo, V. \& Belgiorno, V. Application of electrochemical processes to membrane bioreactors for improving nutrient removal and fouling control. Environmental Science and Pollution Research 24, 321-333 (2016).

31.Ensano, B. M. B. et al.. Combination of Electrochemical Processes with Membrane Bioreactors for Wastewater Treatment and Fouling Control: A Review. Frontiers in Environmental Science 4, (2016).

32.Borea, L. et al. . Are pharmaceuticals removal and membrane fouling in electromembrane bioreactor affected by current density?. Science of The Total Environment 692, 732-740 (2019). 
33.Asif, M. B., M., T. \& Zhang, Z. Electrochemical membrane bioreactors: State-of-the-art and future prospects. Science of The Total Environment 741, (2020).

34.Bani-Melhem, K. Q. Development of a Novel Submerged Membrane Electro-Bioreactor for Wastewater Treatment. (Concordia University, 2008).

35.Shen, L. G. et al.. Membrane fouling in a submerged membrane bioreactor: Impacts of floc size. Chemical Engineering Journal 269, 328-334 (2015).

36.Khandegar, V. \& Saroha, A. K. Electrocoagulation for the treatment of textile industry effluent - A review. Journal of Environmental Management 128, 949-963 (2013).

37.Emamjomeh, M. M. \& Sivakumar, M. Review of pollutants removed by electrocoagulation and electrocoagulation/flotation processes. Journal of Environmental Management 90, 1663-1679 (2009).

38.Ensano, B. M. B. et al.. Applicability of the electrocoagulation process in treating real municipal wastewater containing pharmaceutical active compounds. Journal of Hazardous Materials 361, 367-373 (2019).

39.Ibeid, S. Enhancement of the submerged membrane electro-bioreactor (SMEBR) for nutrient removal and membrane fouling control. (Concordia University, 2011).

40.Castrogiovanni, F. et al. Wastewater treatment and emerging contaminants removal in electro membrane bioreactor using self-forming dynamic membranes. in 16th International Conference on Environmental Science and Technology (2019). 


\section{Figure Captions}

Figure 1. Blue Earth world with dripping water on two hand at wait on abstract black backgound

Figure 2. Illustrazione schematica del fouling reversibile, irreversibile e irrecuperabile in MBR ${ }^{18}$

Figure 3. Illustrazione schematica della formazione iniziale dello strato di cake ${ }^{24}$

Figure 4. Rappresentazione schematica dei processi elettrochimici in un e-MBR ${ }^{33}$

Figure 5. Caratteristiche progettuali dell'impianto e-SFDMBR a scala pilota 


\section{Figures}

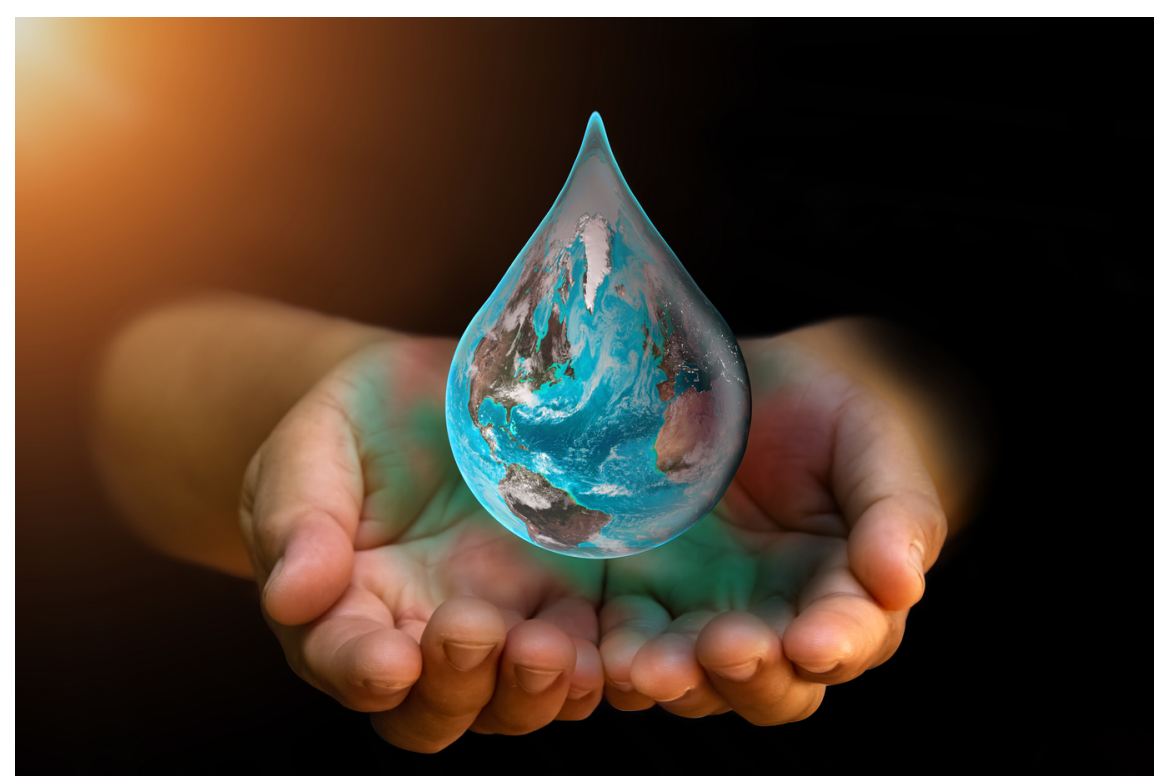

Figure 1: Blue Earth world with dripping water on two hand at wait on abstract black backgound 


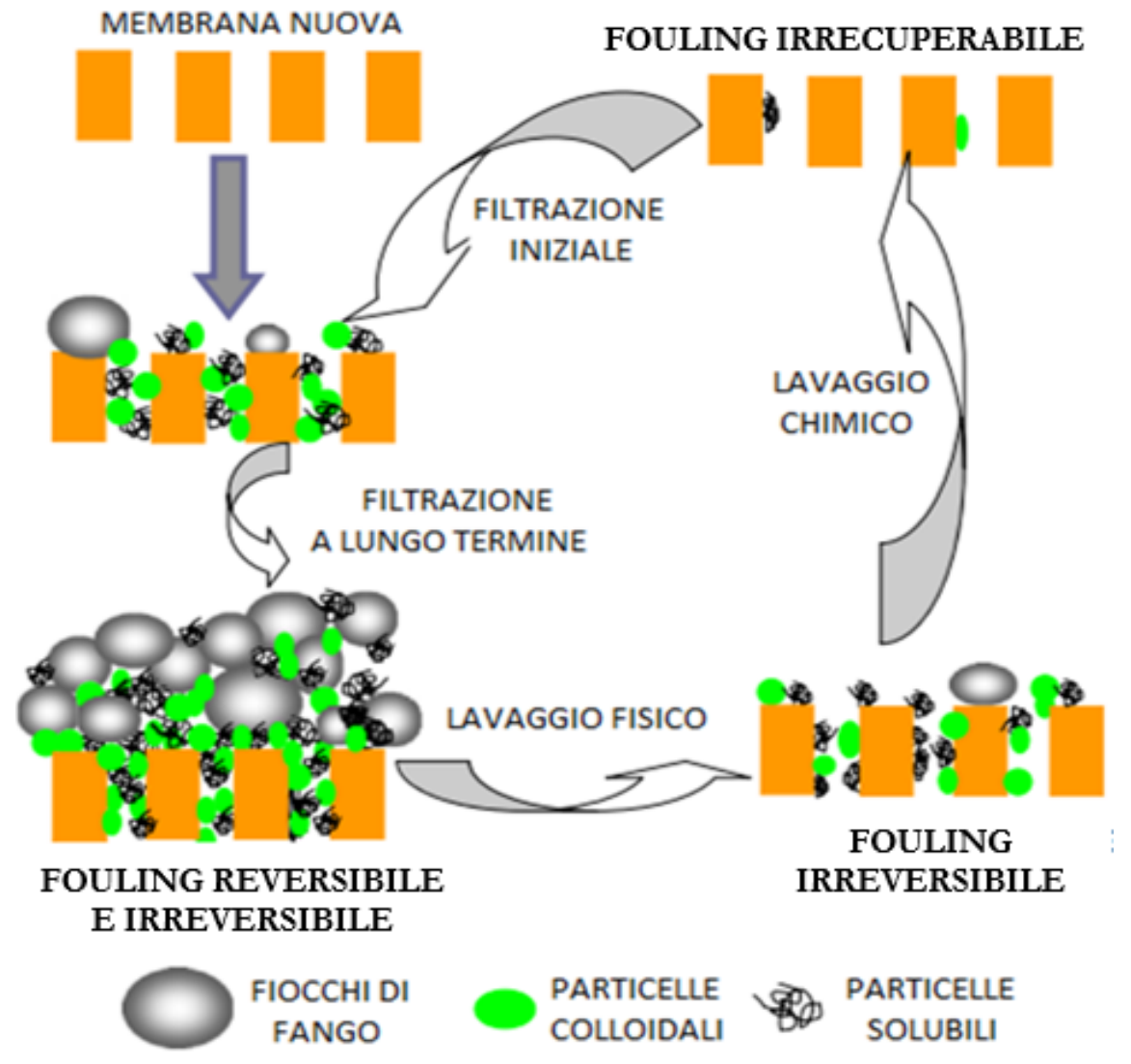

Figure 2: Illustrazione schematica del fouling reversibile, irreversibile e irrecuperabile in MBR ${ }^{18}$ 

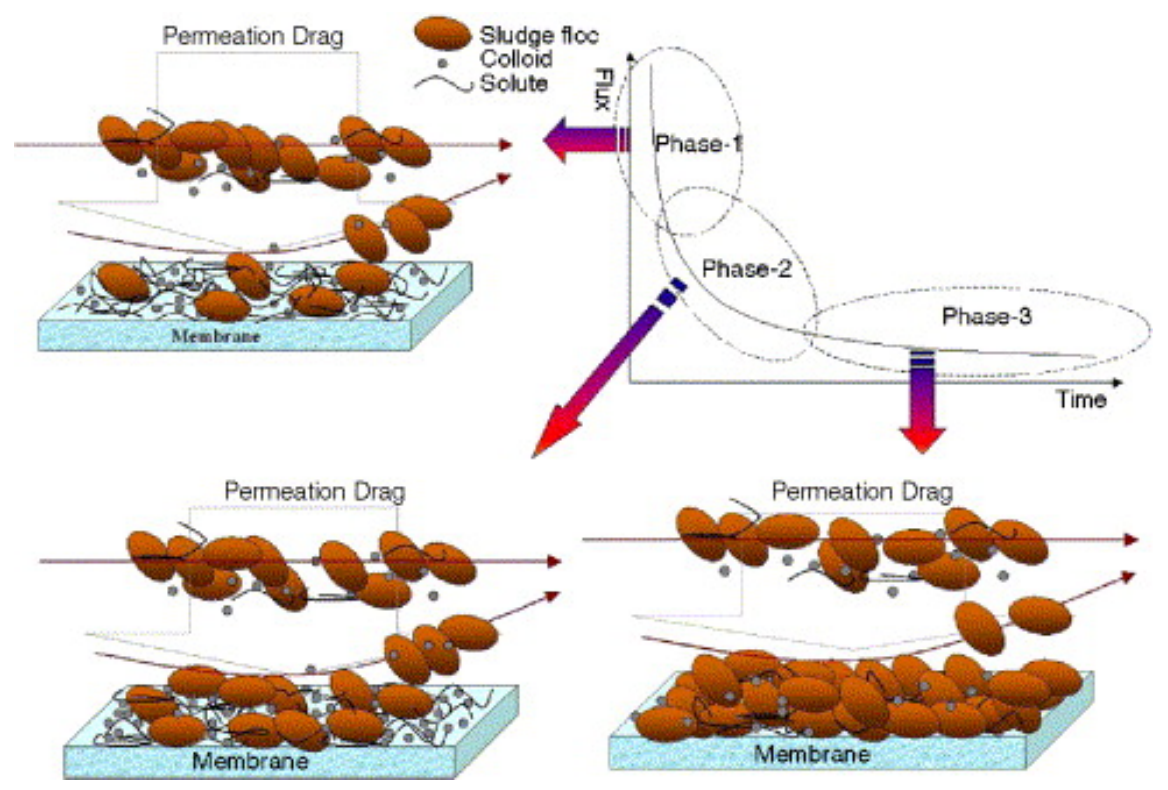

Figure 3: Illustrazione schematica della formazione iniziale dello strato di cake ${ }^{24}$

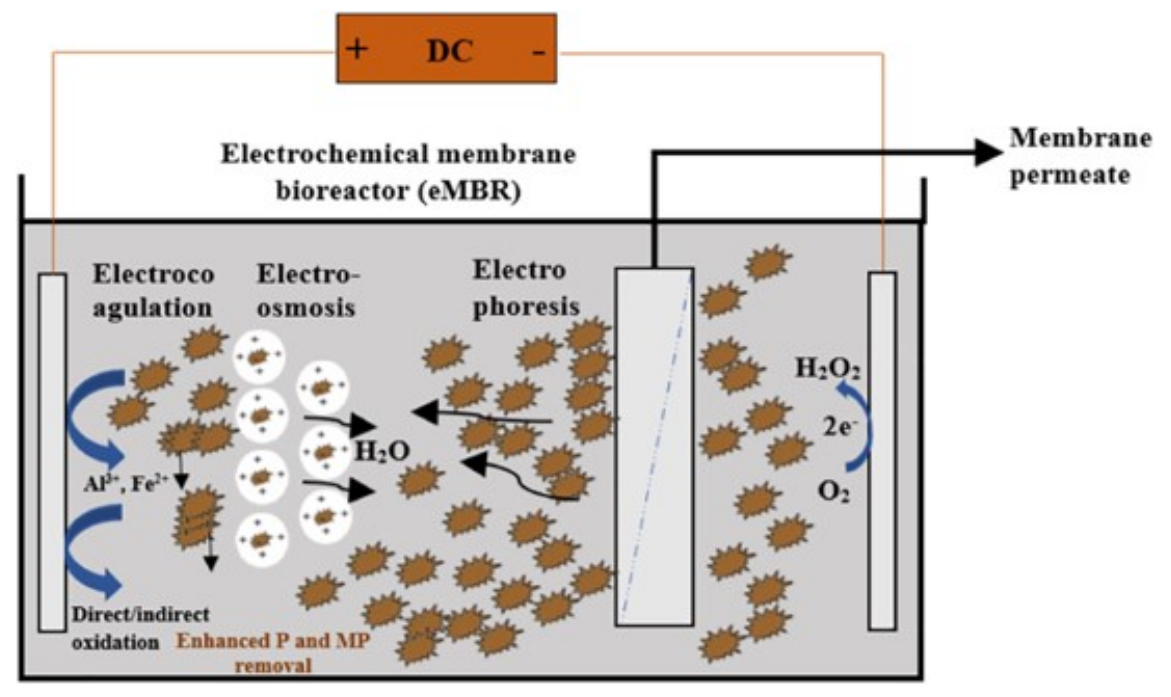

Figure 4: Rappresentazione schematica dei processi elettrochimici in un e-MBR ${ }^{33}$ 


\begin{tabular}{|c|c|}
\hline \multicolumn{2}{|c|}{ CARATTERISTICHE PROGETTUALI } \\
\hline Volume di lavoro & $100 \mathrm{~L}$ \\
\hline Flusso di filtrazione & $30 \mathrm{~L} / \mathrm{m} 2 \mathrm{~h}$ \\
\hline Portata influente & $46,65 \mathrm{~L} / \mathrm{d}$ \\
\hline Portata effluente & $69,98 \mathrm{~L} / \mathrm{d}$ \\
\hline Portata di controlavaggio & $23,32 \mathrm{~L} / \mathrm{d}$ \\
\hline Distanza tra gli elettrodi & $6 \mathrm{~cm}$ \\
\hline Densità di corrente & $0,5 \mathrm{~mA} / \mathrm{cm} 2$ \\
\hline Intensità di corrente & $3,75 \mathrm{~A}$ \\
\hline Ciclo di corrente applicata & $20 \mathrm{~min} \mathrm{OFF} / 5 \mathrm{~min} \mathrm{ON}$ \\
\hline
\end{tabular}

Figure 5: Caratteristiche progettuali dell'impianto e-SFDMBR a scala pilota 